\title{
SUPRA CONDYLAR FEMORAL EXTENSION OSTEOTOMY WITH PATELLAR TENDON ADVANCEMENT TO TREAT FIXED KNEE FLEXION DEFORMITY IN CROUCHING AMBULATORY ADOLESCENTS WITH CEREBRAL PALSY
}

\author{
Hany Mamdouh Hefny, Mahmoud Ali Mahran, Tamer Abd El Meguid Fayyad, \\ and Mohammed Hazem Abd El-Wahab Ahmed
}

Department of Orthopedic Surgery, Faculty of medicine, Ain Shams University.

Cairo , Egypt

Corresponding :

Mohammed Hazem Ahmed, Mobile : 01003726998

E mail:

dr.hazem.zox@gmail.com

Received: $1 / 1 / 2020$

Accepted: 26/1/2020

Online ISSN: 2735-3540

\begin{abstract}
Background: Ambulatory cerebral palsy (CP) patients present with different gait patterns because of muscular spasticity and contractures and subsequent limited range of motion leading to loss of their functional abilities. Crouch gait is one of the most common gait pattern in ambulatory children with $C P$.
\end{abstract}

Aim of the work: The purpose of this study is to evaluate the results of supra condylar femoral extension osteotomy (SCFEO) and patellar tendon advancement (PTA) in the treatment of fixed knee flexion deformity (FKFD) in patients with spastic ambulatory cerebral palsy (CP) to achieve full knee extension and restore an appropriate relationship between quadriceps length and tension in order to maintain knee extension during the stance phase of gait to correct the crouch gait.

Patient and Methods: This prospective case series study was done on 20 patients (37 knees); 12 males and 8 females, who had spastic or mixed (mainly spastic) CP and walked with a crouch gait, and who underwent combined SCFEO and PTA along with the context of single event multi-level surgery.

Results: The mean Fixed Knee Flexion Angle (FKFA) improved from $24.25^{\circ} \pm 11.86^{\circ}$ to be $2.25^{\circ} \pm 2.25^{\circ}$. The mean knee extension lag improved from $15.8^{\circ} \pm 5.68^{\circ}$ to be $4.2^{\circ} \pm 1.31^{\circ}$. The mean Gross Motor Function Measurement (GMFM) improved from 53.3 to be 69.1. The mean Koshino Index (KI) improved from $1.54 \pm 0.29$ to be $1.14 \pm 0.12$.

Conclusion: For crouching adolescents there are 4 questions must be answered: Frist, differentiate between isolated myogenic flexion deformity and combined myogenic-arthrogenic knee flexion deformity. Second, assess isolated myogenic flexion deformity due to hamstring shortening by unilateral popliteal angle test. Third, differentiate between apparent and true hamstring shortening by popliteal shift test; apparent functional hamstring shortening and hamstring lengthening is not indicated. Forth, if the unilateral popliteal angle is positive with negative popliteal shift test, true hamstring shortening is found.

Keywords: Ambulatory cerebral palsy, fixed knee flexion deformity, Supra condylar femoral extension osteotomy, Patellar tendon advancement.

\section{INTRODUCTION:}

Ambulatory cerebral palsy $(\mathrm{CP})$ patients present with different gait patterns because of muscular spasticity and contractures and subsequent limited range of motion leading to loss of their functional abilities. Crouch gait is one of 
the most common gait pattern in ambulatory children with $\mathrm{CP}$ and is contributed to many factors ${ }^{(1)}$.

Rodda et al., described crouch gait as, knee flexion during the stance larger than $20^{\circ}$. The ankle is excessively dorsiflexed and the hip is excessively flexed during stance. The pelvis is in the normal range or tilted posteriorly ${ }^{(2)}$.

Crouch gait often develops and rapidly progresses around the time of the pubertal growth spurt. The development of muscu-loskeletal deformities, collectively referred to as lever arm deformities. The deformities that are frequently seen in adolescents with spastic diplegia are excessive femoral ante version, hip subluxation, patella alta, excessive external tibial torsion, and pes valgus (termed lever arm deformities) $^{(3)}$.

Once a crouch gait has developed, the body mechanics lead to the progression of knee flexion, hip flexion, and ankle dorsiflexion. The loss of an adequate plantar flexion/knee extension couple maintains the ground reaction force behind the knee joint and in front of the hip and ankle joints. Fixed kneeflexion contra-ctures often develop. Patellar tendon elongation and patella alta develop over time and become contributing factors. Knee and back pain have been mentioned as two of the primary factors that limit the walking ability of these patients ${ }^{(4)}$.

Several methods are described in the literature for treatment of fixed knee flexion deformity (FKFD) in CP patients, such as hamstring lengthening, posterior knee capsulotomy and serial casting. Complications such as neurovascular injuries, femoral fractures, skin break-down, recurrence and hyperextension are more often presented with severe deformities. Supra condylar femoral extension osteotomy (SCFEO) has been considered as an alternative to posterior knee capsulotomy for cases in which total extension was not achieved after the soft tissue release ${ }^{(5)}$.

SCFEO was originally described by Osgood in 1913 and has since been variously reported for the treatment of fixed flexion deformities associated with poliomyelitis, myelomeningocele and $\operatorname{arthritis}^{(6)}$.

Patellar tendon advancement (PTA) was originally described by Chandler in 1933 and a modified technique was described by Roberts et $a l^{(7)}$.

Combined SCFEO and PTA have encouraging results regarding pain relief and improvement in community walking levels $^{(4)}$.

\section{AIM OF THE WORK:}

The purpose of this study is to evaluate the results of supra condylar femoral extension osteotomy (SCFEO) and patellar tendon advancement (PTA) in the treatment of fixed knee flexion deformity (FKFD) in patients with spastic ambulatory cerebral palsy (CP) to achieve full knee extension and restore an appropriate relationship between quadriceps length and tension in order to maintain knee extension during the stance phase of gait to correct the crouch gait.

\section{PATIENT AND METHODS:}

\section{Demographics:}

This prospective case series study was done on 20 patients (37 knees); 12 males and 8 females, who had spastic or mixed (mainly spastic) CP and walked with a crouch gait, and who underwent combined SCFEO and PTA along with the context of SEMLS. Their mean age at the time of surgery was 12.50 years (range, 10 to 17 years), the preoperative Gross Motor Function Classification System (GMFCS) was III in 
17 Patients, and II in 3 patients and the mean follow up was 2 years (range 1.5-2.5 years). The study was conducted in Ain Shams University hospitals in the period between March 2016 and September 2018.

\section{$>$ Inclusion criteria:}

1. Confirmed diagnosis of cerebral palsy.

2. Ambulatory adolescents with spastic or mixed hypertonia pattern with predominant spasticity.

3. GMFCS level II- III.

4. Combined myogenic- arthrogenic knee flexion deformity.

5. Fixed knee flexion deformity of $10^{\circ}$ or higher.

\section{Exclusion criteria:}

1. Other progressive neuromuscular disorder.

2. Isolated myogenic knee flexion by examination.

3. Flexion contractures of $>40^{\circ}$.

4. Non communicable patients.
5. Expected non-compliance or no access to facilities of physiotherapy and rehabilitation.

All patients had been given informed consent, and the study protocol was approved by the Institutional Review Board in our university.

Of the 20 patients, involving 37 limbs in twenty cerebral palsy children with crouch gait pattern. All patients had fixed knee flexion deformity and underwent distal femoral extension osteotomy. All patients had concomitant extensor mechanism tightening. All except three patients had bilateral procedures.

The 20 patients were classified according to the expanded and revised GMFCS for children and youth with $\mathrm{CP} .{ }^{(8)}$ Three (15\%) were GMFCS II and seventeen $(85 \%)$ were GMFCS III (Diag. 1). All patients have had bilateral spastic or mixed (mainly spastic) diplegic CP.

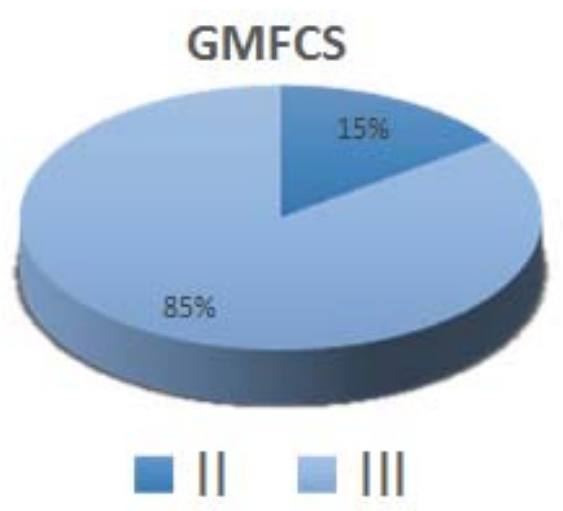

Diag. 1: Pie chart showing the preoperative distribution of GMFCS in the study group.

Methods:

Preoperative evaluation: Patients were evaluated by the following:

\section{History:}

History taking emphasized on, but not limited to, the following points:

- Any recent deterioration of patient activities and anterior knee pain.
- Previous physiotherapy.

- Previous interventions: Most of the patients had previous interventions, either surgery or Botox (BTX) injection, especially of the calf muscles.

\section{Clinical examination:}

Standard couch examination of each patient was done. Goniometric measure- 
ments of joint range of motion and deformities were done, the same way as standards for these examinations. The most important points in physical examination include, but not limited to, the following:

- Hip examination:

- Fixed flexion deformity at the hip by Thomas test.

- Hip range of motion mainly:

o Abduction in extension and flexion for adductor \pm gracilis contracture. o Internal and external rotation, (for femoral anteversion) were measured with the patient prone.

- Knee examination:

- Fixed flexion deformity at the knee:

The supine forced knee extension test (with the hips extended) differentiates between isolated myogenic knee flexion deformity and combined myogenicarthrogenic FKFD (Diag. 2).
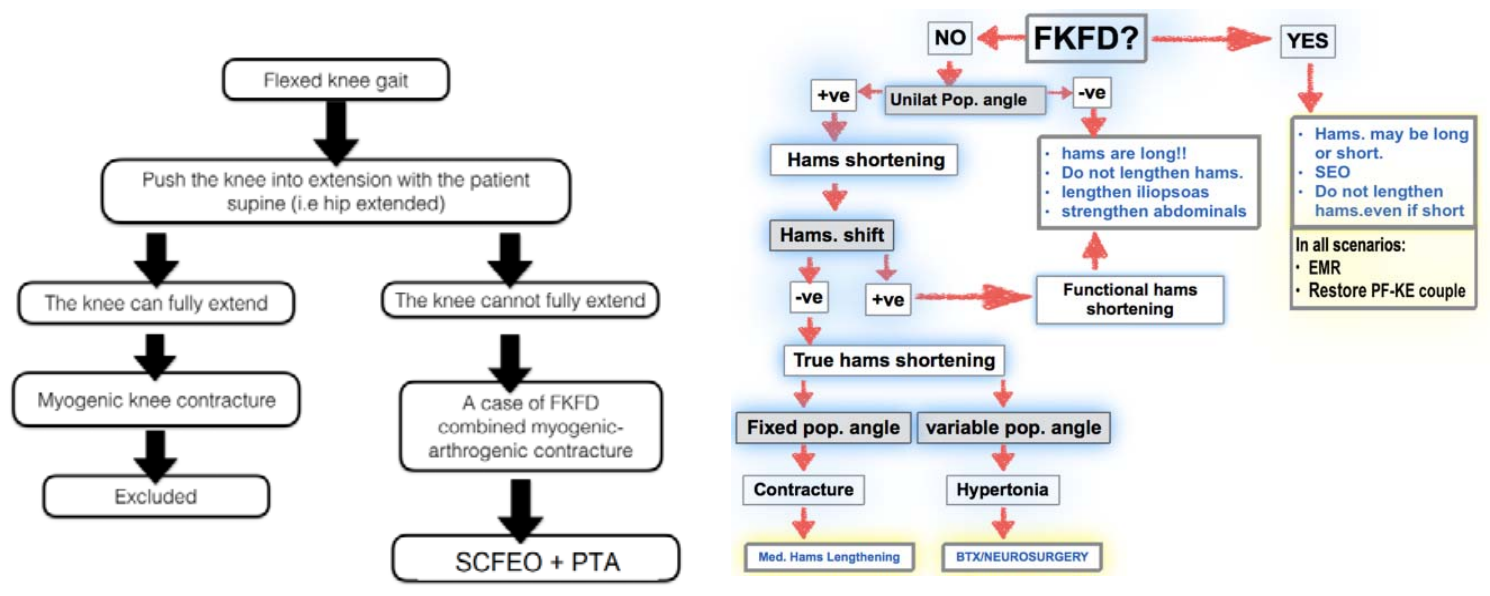

Diag. 2: The knee deformity assessment and management algorithm.

- Hamstring length:

- The popliteal angle.

- The modified popliteal angle.

- Extension lag: The lag to full extension was recorded.

- Prone rectus femoris test by Duncan Ellys' test.

- Ankle and foot examination:

- Measurement of gastrocnemius and soleus length with the use of Silfverskiöld test.

- Tibial torsion: It was measured with the Trans Malleolar Axis method.

- $\quad$ Ankle varus or valgus deformity.

\section{Observational gait analysis:}

Patient was exposed from the foot to the umbilicus leaving underwear rolled to uncover the ASIS anteriorly and PSIS posteriorly. Patient was asked to walk several times along a small corridor and the examiner seated with his eyes at the same level as the patient knees (middle of lower limb). This was done from front and side. Video recording was sometimes done, after consenting, to be visualized several times and in slow motion for more analysis in complex cases.

Evaluation of the existing function and functional needs of the child by GMFM score:

The Gross Motor Function Measure- 88 (GMFM-88) is a standardized observational instrument used to assess motor capacity in CP patients preoperatively and at one year postoperatively $^{(9)}$. 


\section{Radiography:}

- $\quad$ X-ray pelvis AP in neutral hip rotation and frog lateral: two views of the hips were taken for the patients to exclude any hip subluxation or dislocation.

- X-ray both knees AP and dead lateral: dead lateral view was done in knee flexion from $30^{\circ}$ to $90^{\circ}$ and Koshino index (Fig. 1) was calculated to assess the patellar height ${ }^{(10)}$.

- The lateral X-ray of the knee in maximum extension should be reviewed for the degree of knee posterior capsular contracture, patellar height, presence of a stress fracture at the inferior pole of the patella, and the status of skeletal maturation.

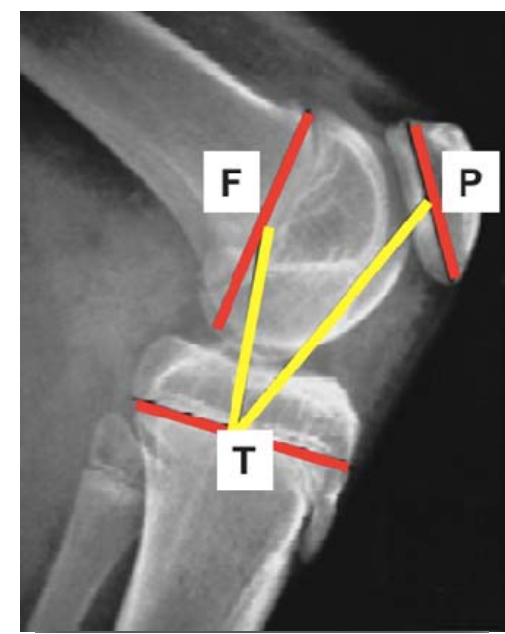

Fig. 1: Lateral knee X-ray of case 8 illustrating Koshino index.

\section{Operative components:}

Anaesthesia: General anaesthesia was used for all patients.

\section{Positioning:}

Supine position allows access for both extension osteotomy and patellar advancement. The procedures are performed under tourniquet control.

\section{Operative technique:}

\section{Approach:}

- The extension osteotomy of the distal femur is performed via a lateral distal femoral incision.

- The patellar advancement is performed through a direct anterior incision centered over the tibial tubercle.

\section{Supra condylar femoral extension osteotomy:}

- The distal femoral physis is located with the aid of fluoroscopy.
- A 4- to 5-cm longitudinal incision is made laterally, with the distal end of the incision at the level of the physis. A subvastus approach is made to the distal femur after incising the iliotibial band. This is most easily accomplished in a distal-to-proximal direction using electrocautery to mobilize the vastus off the intermuscular septum.

- Fluoroscopic imaging is used to localize the osteotomy site (generally $2.5 \mathrm{~cm}$ proximal to the distal femoral physis). The increased healing capacity and increased cross-sectional area in the metaphysis relative to the diaphysis make it very important to perform a metaphyseal osteotomy.

- The level of osteotomy was marked with a saw blade. Another vertical mark is used to mark rotation. The distal osteotomy is performed first. The second osteotomy is performed perpendicular to the femoral shaft, typically meeting the 
first osteotomy at the posterior cortex (although with more severe deformities, a cuneiform wedge including several millimeters of posterior cortex may also be removed to avoid neurovascular stretch) (Fig. 2).

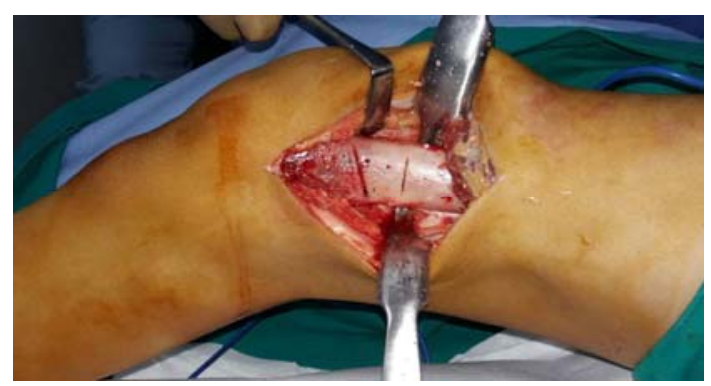

Fig. 2: intraoperative first and second osteotomy

- A large elevator may be used to protect the posterior structures during the osteotomy.

- The anterior wedge of bone is removed.

- The posterior edge of the distal fragment was trimmed to avoid vascular injury.

- The osteotomy was fixed with plate and screws or crossing K-wires depending on the age of the patient and the circumference of the femur (Diag. 3).

- Fixation by smooth K-wires in a crossed configuration. Three pins are usually all that is needed for most children, though two pins are likely to be biomechanically sufficient in many children.

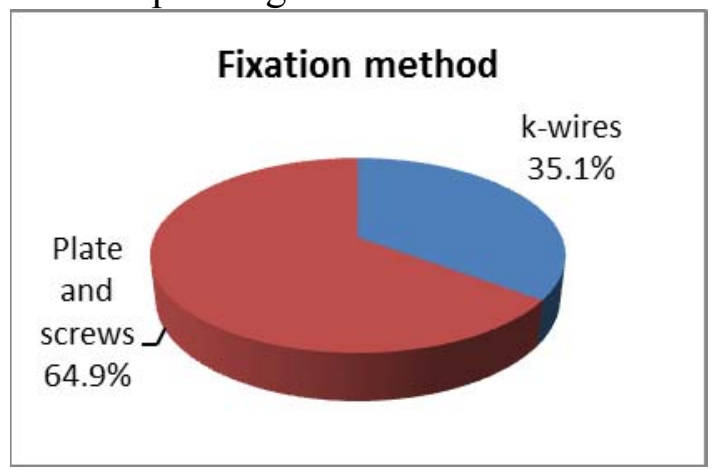

Diag. 3: Pie chart Fixation method distribution of the study group by k-wires (35.1\%), and plate and screws $(64.9 \%)$ of fixation method.

- Fixation by plate, (in our study we use locked proximal humeral plate) (Fig. 3).

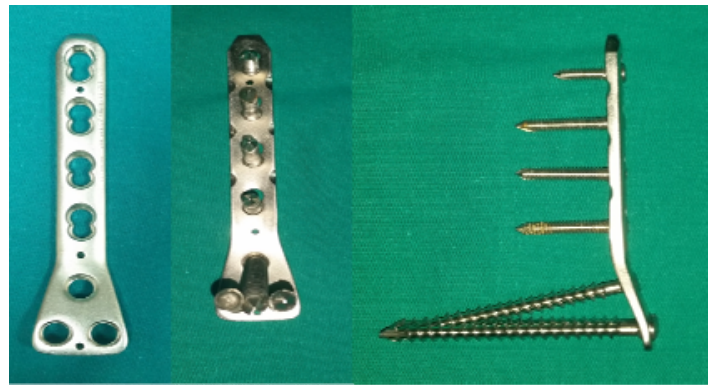

Fig. 3: Shows the locked proximal humeral plate. 
The plate is positioned in the lateral metaphysis with the shaft of the plate parallel to the shin of the tibia and the distal most edge is just 2-3 $\mathrm{mm}$ above the marked physis. According to osteotomy rule 2 , the distal fragment must extend and translate posteriorly to realign the mechanical axis ${ }^{(11)}$. If rotation is planned, the sequence of rotation, translation then angulation is respected to avoid jamming at the osteotomy (Fig. 4).

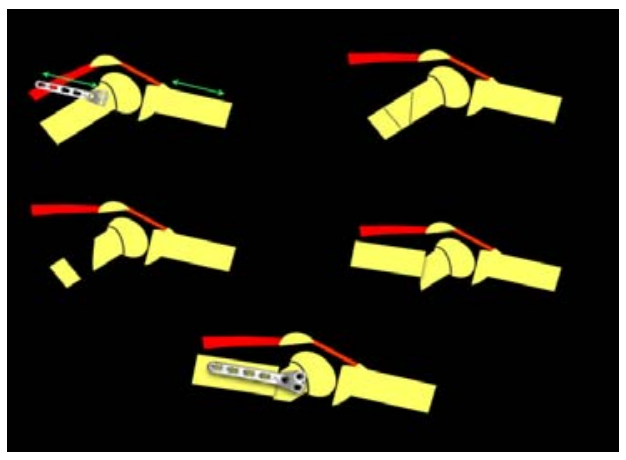

Fig. 4: An anterior based wedge removed with or without femoral shortening with posterior translation of distal fragment.

- Any significant posterior bone prominence should be resected with the oscillating saw.

- The wound is closed in layers.

\section{Extensor mechanism tightening by PTA:}

- Done in all cases using Gillette children's hospital technique. ${ }^{(12)}$

- The patellar tendon is advanced without violating its insertion.

- A T-shaped periosteal incision is made distal to the tibial tubercle apophysis.

- Medial and lateral flaps of periosteum are elevated.

- The tendon is separated from the cartilaginous tibial tubercle apophysis using a fresh scalpel, care must be taken to maintain an adequate thickness of tendon (about $2 \mathrm{~mm}$ ) without defects.
- The next step is placement of a tension band from the patella to the tibia to protect the repair by a nylon tape suture passed by an ACL guide. Sutures are passed along the edges of the patella via a subcutaneous tunnel to the anterior incision (Fig. 5).

- The patella is advanced distally by tensioning the nylon tape until the inferior pole of the patella is at the femoral-tibial joint line, at which point the knot in the nylon tape is tied.

- Two Krackow stitches are placed in the patellar tendon, one medially and one laterally, using Ethibond suture.

- These sutures are tied deeply under the periosteal flaps (Fig. 6).

- The periosteal flaps are then sewn over the patellar tendon (Fig. 7).

- The wound is closed in routine fashion.

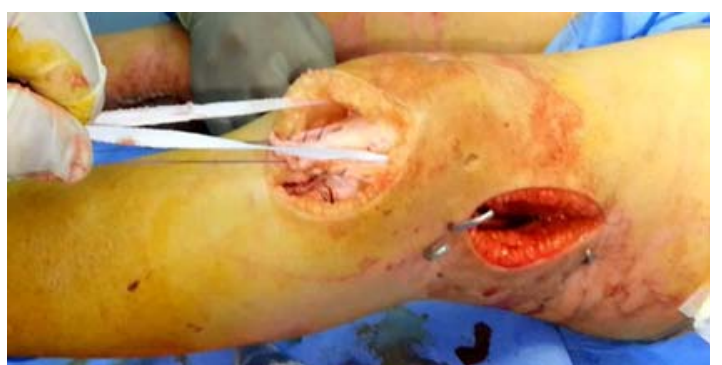

Fig. 5: Naylon tape placed transversely across patella 


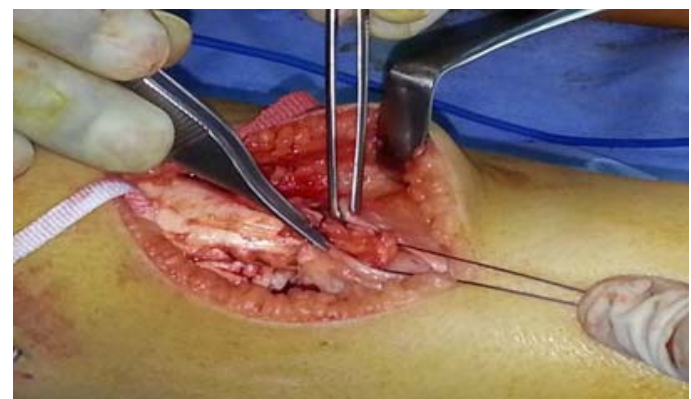

Fig. 6: The patellar tendon is advanced and the periosteal flaps covering it.

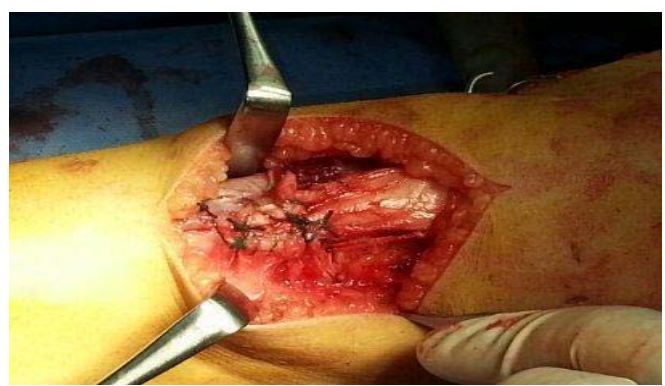

Fig. 7: The patellar tendon is advanced under the periosteal flaps, and the flaps are repaired over the tendon.

Other associated operations were done to correct associated deformities found in the patient lower limbs.

\section{Postoperative components:}

\section{1) Postoperative management:}

- All patients had high above knee cast placed at the time of surgery.

- Anteroposterior and lateral radiographs were done to confirm proper alignment.

\section{2) Postoperative follow up:}

- Patients were reviewed at 2 weeks postoperatively.

- The osteotomies were followed up by serial X-rays till complete healing.

- Six weeks after surgery cast is removed after complete healing of the osteotomy site.

- Postoperative rehabilitation program involves strengthening exercises of the upper limbs and to the unfixed Joints (e.g. hips, toes) as tolerated even before removal of the lower limbs cast.

- An intensive rehabilitation program starts at the earliest radiological evidence of satisfactory callus formation (6-8 weeks). It is prescribed as 5 times/week for six consecutive months for four hours/session. The physiotherapy training is fashioned individually for each child based on his/her functional capacity.

- Immediately after removing the cast, a custom-made knee immobilizers are used to achieve early weight bearing exercises. The ground reaction ankle foot orthosis (GRAFO) is used in patients with weak quadriceps or weak triceps surae to prevent recurring of crouch gait.

- Patients were followed-up by multidisciplinary team at the 6th week, the 12th weeks then every six months till the final follow up. The mean follow up was 2 (range 1.5-2.5) years.

\section{3) Postoperative evaluation:}

- Fixed knee flexion angle.

- Extension lag angle.

- Koshino index.

- GMFM score.

Those parameters were also reevaluated after one year postoperatively. 


\section{Statistical analysis:}

Recorded data were analyzed using the statistical package for social sciences, version 20.0 (SPSS Inc., Chicago, Illinois, USA). Quantitative data were expressed as mean \pm standard deviation (SD). Qualitative data were expressed as frequency and percentage.

\section{The following tests were done:}

- $\quad$ Paired sample t-test of significance was used when comparing between related samples.

- Chi-square $\left(\mathrm{x}^{2}\right)$ test of significance was used in order to compare proportions between qualitative parameters.

- The confidence interval was set to $95 \%$ and the margin of error accepted was set to $5 \%$.

\section{RESULTS}

\section{Statistical analysis:}

All parameters were measured preoperatively and at 1 year postoperative. The mean operative time of the supra condylar femoral extension osteotomy and patellar tendon advancement was $60 \pm 30$ minutes (range, 30 to 180). The mean blood loss was $100 \mathrm{~mL}$ (range, 50 to $300 \mathrm{~mL}$ ).

\section{[A] Clinical results:}

\section{(1) Fixed Knee Flexion Angle (FKFA):}

At the one year follow up, the mean FKFA was $2.25^{\circ} \pm 2.25^{\circ}$ (range $0-15$ ) that is compared with the mean preoperative FKFA of $24.25^{\circ} \pm 11.86^{\circ}$ (range 15-40) (Table 1).

Table (1): The extent of the difference over the periods through FKFA in the study group.

\begin{tabular}{|l|c|c|c|c|c|}
\hline \multirow{2}{*}{ Period } & \multirow{2}{*}{ FKFA } & \multicolumn{3}{|c|}{ Paired Sample t-test } \\
\cline { 3 - 6 } & & Mean Diff. & $\begin{array}{c}\text { Delta } \\
\text { change }\end{array}$ & t-test & p-value \\
\hline Pre-operative & $24.25 \pm 11.86$ & & & & \\
\hline Post-operative & $7.68 \pm 3.19$ & -16.57 & -0.68 & 5.471 & $<0.001 * *$ \\
\hline After 1 year & $2.25 \pm 2.25$ & -22 & -0.91 & 7.734 & $<0.001 * *$ \\
\hline
\end{tabular}

Postoperative and after 1 year ( $t=5.313$, with $p$-value $\left.<0.001^{* *}\right)$

p-value $<0.001 \mathrm{HS}^{* *}$

This table shows highly statistically significant decrease mean of postoperative and after 1 year compared to preoperative according to FKFA.

\section{(2) Extension Lag Angle:}

The mean knee extension lag at the one year follow up was $4.2^{\circ} \pm 1.31^{\circ}$ (range 5-25) compared with mean preoperative knee extension lag of $15.8^{\circ} \pm 5.68^{\circ}$ (range $15-30$ ) (Table 2).

Table (2): The extent of the difference over the periods through extension lag angle in the study group.

\begin{tabular}{|l|c|c|c|c|c|}
\hline \multirow{2}{*}{\multicolumn{1}{|c|}{ Period }} & \multirow{2}{*}{ Extension lag angle } & \multicolumn{4}{|c|}{ Paired Sample t-test } \\
\cline { 3 - 6 } & & $\begin{array}{c}\text { Mean } \\
\text { Diff. }\end{array}$ & $\begin{array}{c}\text { Delta } \\
\text { change }\end{array}$ & t-test & p-value \\
\hline Pre-operative & $15.79 \pm 5.68$ & & & & \\
\hline Post-operative & $8.19 \pm 3.61$ & -7.60 & -0.48 & 4.197 & $<0.001^{* *}$ \\
\hline After 1 year & $4.21 \pm 1.31$ & -11.58 & -0.73 & 6.447 & $<0.001^{* *}$ \\
\hline
\end{tabular}

Postoperative and after 1 year ( $t=4.546$, with $p$-value $\left.0.021^{*}\right)$

p-value $<0.001 \mathrm{HS}^{* * *}$

This table shows highly statistically significant decrease mean of postoperative and after 1 year compared to preoperative according to extension lag angle. 
(3) Gross Motor Function Measurement (GMFM):

The mean GMFM at the one year postoperative follow up was 69.1(range
57.80 - 83.70) compared with mean preoperative GMFM of 53.3(range 44.80 64.70) (Diag. 4).

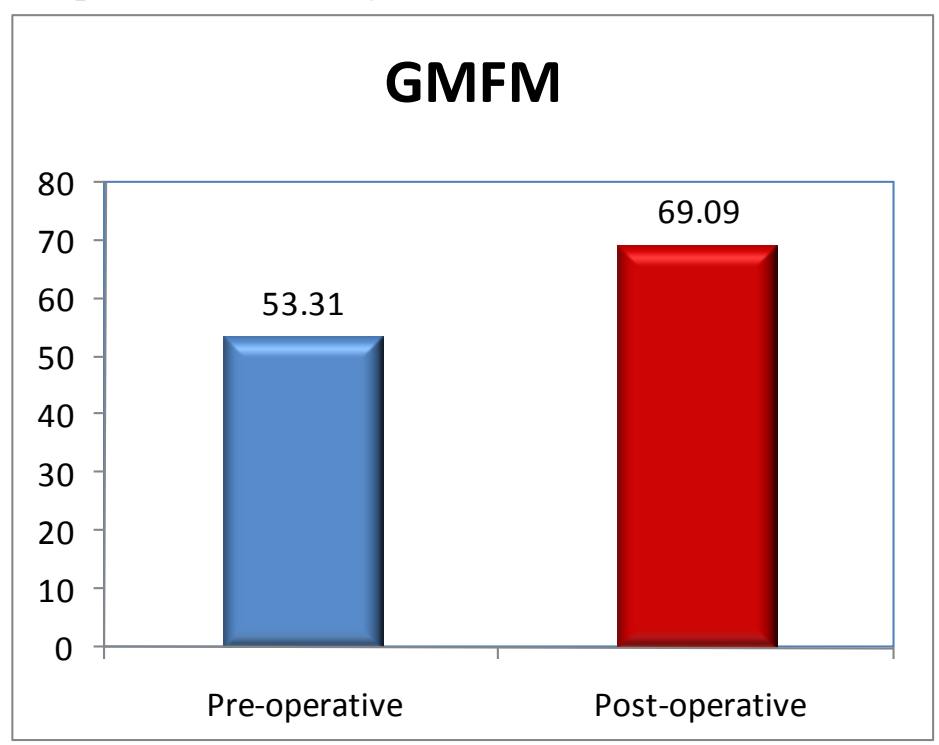

Diag. 4: Bar chart between preoperative and one year postoperative according to GMFM.

(4) Gross Motor Function classification system:

Preoperatively 3 patients were GMFCS level II and 17 patients were GMFCS level
III, however 15 patients were found to be GMFCS level II and 5 patients were found to be GMFCS level III at the one year postoperative follow up (Table 3 ).

Table (3): Comparison between preoperative and one year postoperative according to GMFCS.

\begin{tabular}{|l|c|c|c|c|}
\hline GMFCS & Pre-operative & $\begin{array}{c}\text { One year Post- } \\
\text { operative }\end{array}$ & \multicolumn{2}{|c|}{ Chi-square test } \\
\cline { 1 - 1 } & & $\mathrm{x}^{2}$ & $\mathrm{p}$-value \\
\hline II & $3(15 \%)$ & $15(75 \%)$ & 12.222 & $0.005^{*}$ \\
\hline
\end{tabular}

$x^{2}$ : Chi-square test; $p$-value $<0.05 S^{*}$

This table shows statistically significant difference between preoperative and postoperative according to GMFCS.

[B] Radiological results by Koshino

The mean KI at the one year follow up was $1.14 \pm 0.12$ (range $1.0-1.30$ ) compared with mean preoperative KI of $1.54 \pm 0.29$ (range $1.38-2.14$ ) (Table 4). Index:

Table (4): The extent of the difference over the periods through KI in the study group.

\begin{tabular}{|l|c|c|c|c|c|}
\hline \multirow{2}{*}{\multicolumn{1}{|c|}{ Period }} & \multirow{2}{*}{ Koshino index } & \multicolumn{4}{c|}{ Paired Sample t-test } \\
\cline { 3 - 6 } & & $\begin{array}{c}\text { Mean } \\
\text { Diff. }\end{array}$ & $\begin{array}{c}\text { Delta } \\
\text { change }\end{array}$ & t-test & p-value \\
\hline Pre-operative & $1.54 \pm 0.29$ & & & & \\
\hline Post-operative & $1.03 \pm 0.10$ & -0.51 & -0.33 & 7.435 & $<0.001^{* *}$ \\
\hline After 1 year & $1.14 \pm 0.12$ & -0.40 & -0.26 & 5.700 & $<0.001^{* *}$ \\
\hline
\end{tabular}

Postoperative and after 1 years ( $t=3.149$, with $p$-value $\left.0.003^{*}\right)$

p-value $<0.001 \mathrm{HS}^{* *}$ 
This table shows highly statistically significant decrease mean of the one year postoperative compared to preoperative according to KI.

\section{[D] Complications:}

In this series, heel ulcer from the cast occurred 3 times, which resolved with frequent dressing and off-loading for 4 weeks. Recurrence of 5 degrees of flexion occurred in 3 patients (5 limbs). Anterior pelvic tilt was noticed in 3 patients ( 5 limbs) as well (Table 5).

Table (5): Complications distribution of the study group.

\begin{tabular}{|l|c|c|}
\hline Complications & No. & $\%$ \\
\hline Ulcer from the cast & 3 & $8.1 \%$ \\
\hline Anterior pelvic Tilt & 5 & $13.5 \%$ \\
\hline Recurrence of 5 degrees of flexion & 5 & $13.5 \%$ \\
\hline No Complications & 24 & $64.9 \%$ \\
\hline Total & 37 & $100.0 \%$ \\
\hline
\end{tabular}

This table shows that the Ulcer from the cast $(8.1 \%)$, Anterior pelvic Tilt $(13.5 \%)$, Recurrence (13.5\%) and No Complications $(64.9 \%)$ of complications.
There is a relation between anterior pelvic tilt and hamstring lengthening as it was noticed in patients done hamstring lengthening (Table 6).

Table (6): Relation between anterior pelvic tilt and hamstring lengthening.

\begin{tabular}{|c|c|c|c|c|c|c|}
\hline \multirow[t]{3}{*}{ Anterior pelvic Tilt } & \multicolumn{4}{|c|}{ Hamstring lengthening } & \multirow{2}{*}{\multicolumn{2}{|c|}{ Total }} \\
\hline & \multicolumn{2}{|c|}{ Medial hamstring } & \multicolumn{2}{|c|}{$\begin{array}{l}\text { No hamstring } \\
\text { lengthening }\end{array}$} & & \\
\hline & No. & $\%$ & No. & $\%$ & No. & $\%$ \\
\hline Yes & 5 & $38.5 \%$ & 0 & $0.0 \%$ & 5 & $13.5 \%$ \\
\hline No & 8 & $61.5 \%$ & 24 & $100.0 \%$ & 32 & $86.5 \%$ \\
\hline Total & 13 & $100.0 \%$ & 24 & $100.0 \%$ & 37 & $100 \%$ \\
\hline Chi-square test & \multicolumn{6}{|c|}{7.636} \\
\hline$p$-value & \multicolumn{6}{|c|}{$0.006^{*}$} \\
\hline
\end{tabular}

This table shows statistically significant relation between anterior pelvic tilt according to hamstring lengthening as it shows increase in anterior pelvic tilt incidence in cases where hamstring lengthening was done.

\section{CASE PRESENTATION:}

Preoperative data: Male 15 years old with GMFCS grade III, Spastic diplegic with history of hypoxic, encephalopathy and incubator, Flexion angle $40^{\circ}$, Extension lag $30^{\circ}$, Patella alta with KI 1.92, GMFM score was $47.6 \%$.

Operative data: Patient had supracondylar extension osteotomy, PTA, Bilat. iliopsoas, adductor, med. Hamstring and lat. column lengthening with tibialis post advancement. Intraoperative compli-cation none. Bleeding about $50 \mathrm{cc}$. Implant used plate and screws.

Postoperative follow up: Above knee cast was applied for 6 weeks with knee in full extension. Patient was reviewed at 2 weeks postoperatively, then after 4 weeks for cast removal and then was reviewed monthly in 1st three months then at three months interval. Six weeks after surgery cast was removed and rehabilitation started. Fixed knee flexion angle become $7^{\circ}$ and GMFM score become61.2\%. Extension lag become $10^{\circ}$ with KI 1.1 
Hany Mamdouh Hefny, et al.,

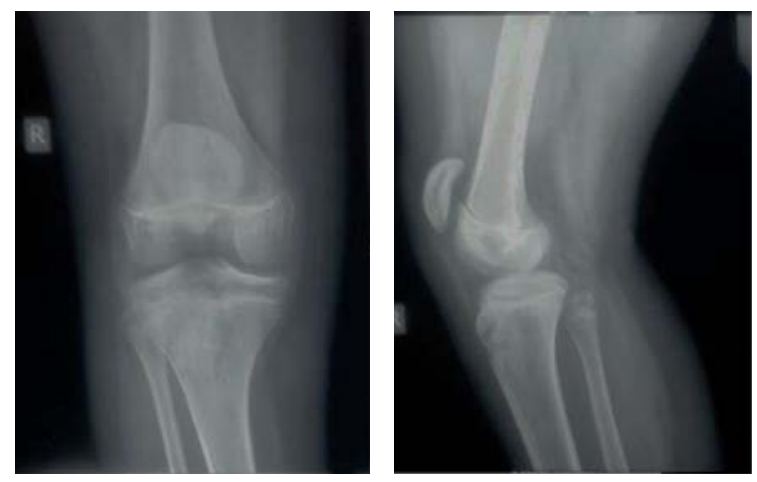

Fig. (8): Preoperative x-ray.

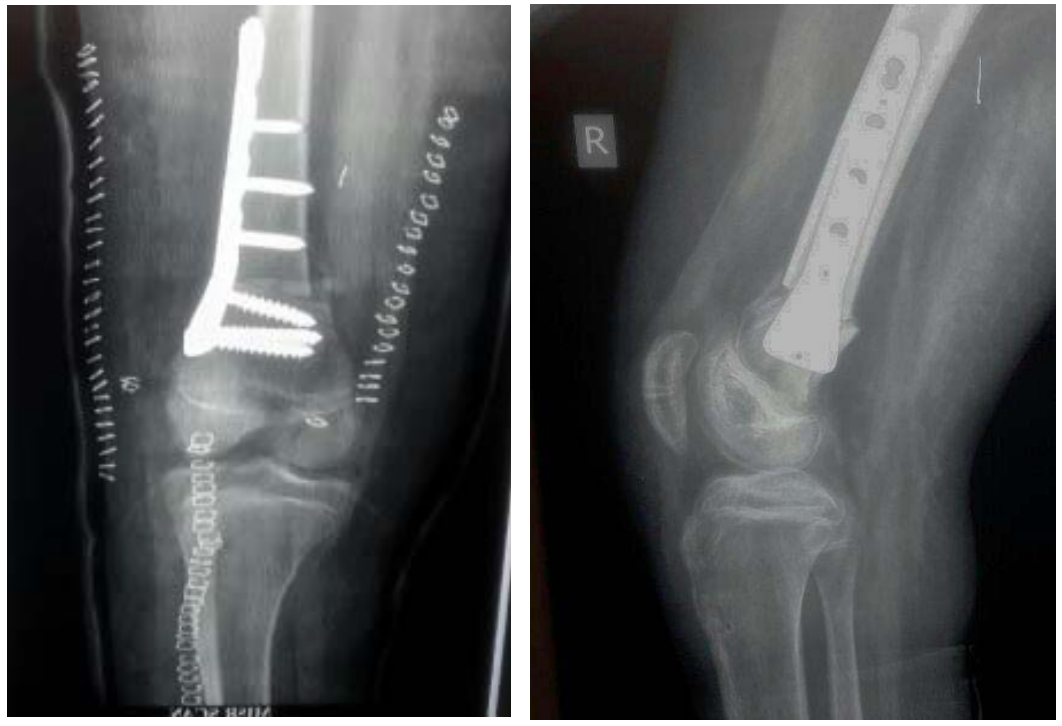

Fig. (9): 6 weeks postoperative.
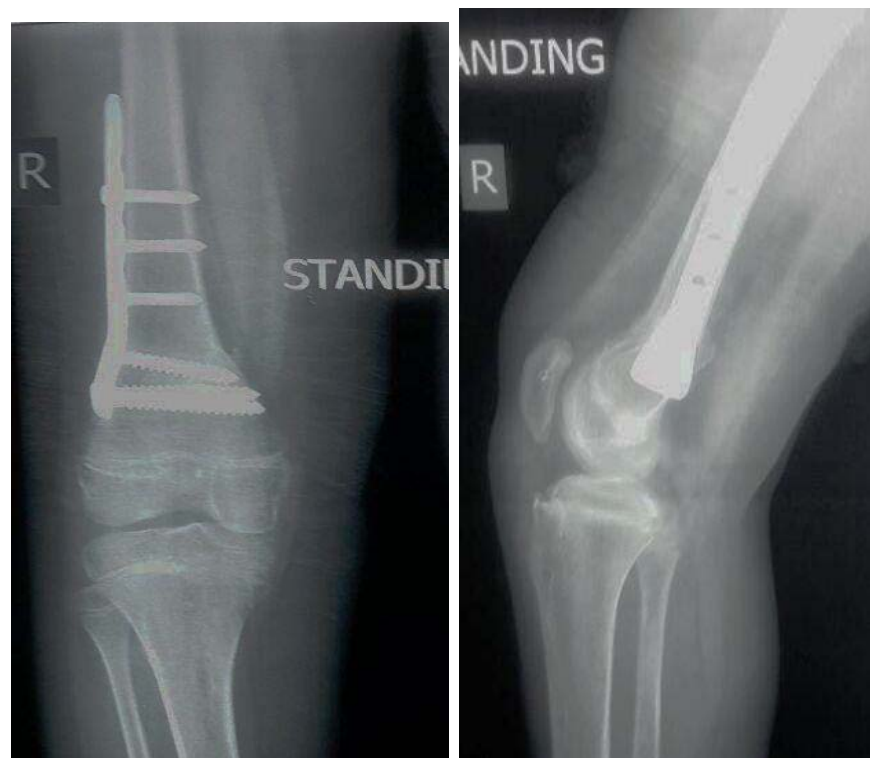

Fig. (10): one year postoperative. 


\section{DISCUSSION:}

In this study, we categorize the pathology of knee flexion into 2 main types; the myogenic type and the combined myogenic-arthrogenic type. A long standing isolated myogenic knee flexion deformity will eventually progress into a combined myogenic-arthrogenic fixed knee flexion deformity as the knee posterior capsule contracts and femoral condyles flattens due to the adaptive bony changes. Preoperative clinical assessment differentiates between the two types. As each type needs a different procedure for correction (Diag. 2).

In literature, various procedures were described for correcting FKFD in general without paying attention to differentiation between both types.

Temelli et al., Gage JR, Westberry et al., and Beals et al. ${ }^{(13-16)}$ attempted hamstring lengthening and serial casting alone or combined with posterior knee capsulotomy; Isolated muscle lengthening procedure can correct the myogenic static type . However, with the combined type, it cannot even correct a FKFD as small as 5 to 10 degrees. In higher flexion degrees, acute correction after combined procedures (hamstring lengthening and posterior capsulotomy) for the combined type, increases the risk of neurovascular stretch injury therefore necessitating additional gradual correction by serial casting. The authors believe that in those cases, additional extensor mechanism tightening procedure has to be deferred to a later stage, when maximum extensor mechanism redundancy is reached with full knee extension in order to achieve a proper tension without compromising its repair.

Stout et al., Filho et al., Novacheck et al., and Rutz et al. ${ }^{(4,5,17-18)}$ described SCFEO for correcting FKFD of $40^{\circ}$ or less, as part of SEMLS.
Posterior cruciate ligament (PCL) is an integral part of the posterior knee capsule and it becomes pathologically contracted as part of posterior capsular contracture. Gavriliu et al. ${ }^{(19)}$ believe that in long standing combined myogenic-arthrogenic FKFD it becomes increasingly difficult to stretch a contracted PCL unless it is incised along with the posterior knee capsule. PCL transection can compromise postero-lateral knee stability and may result in full extension with posterior tibial sublaxation, which is something undesirable in a neurologically compromised child. ${ }^{(19)}$ Additionally, in older children with long standing deformity, bony changes will resist correction by posterior capsulotomy alone. $^{(22)}$

Young et al., and Klatt and Stevens ${ }^{(20,21)}$ described guided growth as an alternative to posterior knee capsulotomy in the combined type. This procedure needs enough remaining time before physeal closure with unpredictable results in adolescents. Moreover, it cannot correct associated femoral rotational deformities and its gradual nature cannot permit proper simultaneous extensor mechanism retightening procedure.

Filho et al. ${ }^{(5)}$ reported reduction in the mean FKFD angle from $15.91^{0}$ to $5.68^{0}$. Their preoperative evaluation did not provide a clear clinical plan to differentiate between combined myogenic-arthrogenic and isolated myogenic knee flexion contracture elements. Their high recurrence rate of $22.7 \%$ and the excessive anterior pelvic tilting are explained by the fact that they didn't attempt extensor mechanism retightening and attempting to lengthen hamstrings (if they weren't already lengthened), respectively. Rutz et al. ${ }^{(18)}$ didn't mention the complication of excessive anterior pelvic tilting which we believe must have occurred.

In agreement with Healy et al. ${ }^{(22)}$, we believe that in such scenario, hamstrings 
will be the only protective structure against sciatic stretch and that lengthening them will lead to high chance of sciatic injury, especially in higher flexion degrees. In such clinical scenario, we recommend femoral shortening with extension. This will decrease sciatic tension but again this will cause a relative hamstring lengthening and might precipitate an excessive anterior pelvic tilting.

Filho et al. and Rutz et al. ${ }^{(5,18)}$ regarded SCFEO as a salvage procedure after failure of hamstring lengthening. It is worth mentioning that SCFEO cannot be regarded as a salvage operation. Rather, it has a specific indication in the combined myogenic-arthrogenic type which cannot be corrected by hamstring lengthening. Combining SCFEO with simultaneous or prior hamstring lengthening may cause excessive anterior pelvic tilting. ${ }^{(5,2)}$ In their study, Healy et al. ${ }^{(22)}$ found that both hamstring length and velocity increase substantially after extension without simultaneous hamstring lengthening .

Long standing walking in crouch pattern has been shown to cause progressive stretching of the Extensor mechanism with patella alta, oblong patella and tibial traction apophysitis. This will cause a clinically detectable extensor lag and will precipitate recurrence of crouch pattern. ${ }^{(4,7,17)}$ Accordingly, extensor mechanism re-tensioning should be regarded as a cardinal procedure to be done simultaneously with SCFEO.

Our study recommends combining SCFEO and PTA for correcting FKFD. It shows highly significant improvement in all the outcome measurements. It is a safe and effective operation that can correct up to 40 degrees of what we call the combined myogenic-arthrogenic FKFD. ${ }^{(4)}$ In addition, femoral rotational deformities could be corrected from the same osteotomy. Simultaneous extensor mechanism retensioning has been shown to improve knee extension power and reduce recurrence rate $^{(4,7)}$.

Novacheck et al. $^{(17)}$ reported the first detailed technique of combined SCFEO and PTA in crouching patients. However, they lengthened hamstrings when deemed tight intra-operatively and all patients experienced anterior pelvic tilting.

In our study $38.5 \%$ of the cases that have done hamstring lengthening prior our operation experienced anterior pelvic tilting. On the other hand, none of the patients who didn't have their hamstring lengthened developed anterior pelvic tilt.

We believe that differentiating between isolated myogenic and combined myogenicarthrogenic knee flexion contracture is mandatory before intervention to decide between hamstring lengthening and SCFEO. When SCFEO is indicated, simultaneous hamstring lengthening should not be attempted to avoid excessive anterior pelvic tilting.

We routinely tensioned the extensor mechanism and the mean extension lag improved from 15.8 to 4.2 degrees at the latest follow up.

In our study, we experienced recurrence of 10 degrees of flexion in 3 cases despite PTA; and it is thought to be due to the ability of the growth plate to remodel in younger age.

Joseph et al. ${ }^{(23)}$, described combining femoral shortening only osteotomy and extensor mechanism re-tensioning. Extension at the osteotomy was not attempted except in 2 osteotomies. This is followed by a second stage of both lateral and medial hamstring lengthening with semitendinosus transfer, to avoid excessive anterior pelvic tilting.

We believe that shortening osteotomy alone without extension at the osteotomy level in FKFD cannot achieve full knee extension unless, theoretically, performed 
distal to the femoral attachment of knee capsule. Moreover, even in cases of myogenic static knee flexion when hamstring lengthening is indicated, combining medial and lateral hamstring is not recommended in literature due to high incidence of resultant knee recurvatum in stance phase of gait ${ }^{(20)}$.

In our study, we attempted femoral shortening with extension in few cases of higher flexion degrees $(>30)$, when we felt excessive posterior tension intraoperatively. We believe it resulted in excessive anterior pelvic tilt in some cases.

SCFEO has specific indications and should not be considered a final salvage procedure. This study is to document specific indication for the SCFEO and PTA. Clinically differentiating between isolated myogenic and combined myogenicarthrogenic elements is important preoperatively and intra-operatively under anesthesia; in a FKFD, a combined myogenic-arthrogenic element is present and warrants an extension osteotomy without hamstring lengthening. Extensor mechanism re-tensioning is an essential part of the procedure that should be done to prevent recurrence and to improve the extension lag. This is to be done in the same operative session after achieving full limb extension to guarantee the maximum possible tension and secure reattachment of the extensor mechanism.

A considerable majority of studies on major orthopedic interventions in $\mathrm{CP}$ children are retrospective. ${ }^{(24-25)}$ Generally, retrospective studies are known for their defective capability of controlling bias and confounding variables. (24-25) We believe that the prospective nature of our study adds strength to the results. Outcome measures for single event multilevel surgery in crouching $\mathrm{CP}$ children include a wide variety of objective and subjective assessment tools. $^{(26)}$
A unique feature of this study is using the GMFM- 88 score as outcome measure to detect changes in gross motor function in patients with $\mathrm{CP}$ undergoing combined SCFEO and PTA. All cases show significant improvement in their mean preoperative GMFM of 53.31 that became 69.09 postoperatively.

As regard the complications in this series, ulcer from the cast was noticed 3 times and was managed by frequent dressing and off-loading for 4 weeks. Recurrence of 5 degrees of flexion occurred in 5 limbs. There was no sciatic nerve stretch injuries in our study.

In this study concomitant hamstrings lengthening was not done in order to leave hamstrings intact in order to protect the sciatic nerve from stretch injury with the acute correction, and crouch gait improved without anterior pelvic tilting. Femoral shortening was done with higher flexion degrees when excessive posterior soft tissue tension was clinically detected intraoperatively, accepting the resultant excessive anterior pelvic tilting.

The strength of the study was that the same multi-disciplinary team assessed almost all of the patients over the whole study period, using GMFM-88 and observational gait analysis.

Another strength was that the patients were evaluated with different outcome measures. We have not found any previous studies with such broad outcome evaluation.

Our study had certain limitations. The number of children was small, and there was considerable variation in age at operation and in the functional levels of the patients. The type and number of previous operations and combinations of surgical procedures made it difficult to evaluate the effects of a specific procedure. Because we had no control group, and changes in gait function cannot be caused by surgery alone. In this study the quality of life assessment 
instruments were not used. In addition, instrumented gait analysis was not used. We acknowledge such a study limitation.

\section{Conclusion:}

For crouching adolescents there are 4 questions must be answered:

Frist, differentiate between isolated myogenic flexion deformity and combined myogenic-arthrogenic knee flexion deformity by multidisciplinary preoperative assessments and examination under general anesthesia.

In combined myogenic-arthrogenic SCFEO is done. Extensor mechanism retensioning is an integral part of the procedure reduced to recurrence rate and to improve knee extension strength.

Hamstring lengthening should not be attempted in cases of combined myogenicarthrogenic FKFD. Instead of that a femoral shortening osteotomy could be done, which results in a relative hamstring lengthening that might cause excessive anterior pelvic tilting, but on the expense of protecting the neurovascular structures from excessive tension.

Second, if there is no combined myogenic-arthrogenic flexion deformity we should assess isolated myogenic flexion deformity due to hamstrings by unilateral popliteal angle test.

Third, in cases of positive unilateral popliteal angle test, we should differentiate between apparent and true hamstring shortening by popliteal shift test; if the popliteal shift test is positive, that is apparent functional hamstring shortening and hamstring lengthening is not indicated.

Forth, if the unilateral popliteal angle is positive with negative popliteal shift test, true hamstring shortening is found. Hamstring contracture and hypertonia should be differentiated from each. Hamstring lengthening is done in cases of muscle contracture. On the other hand, BTX injection and tone management in cases of hypertonic spastic hamstrings.

\section{REFERENCES}

1. Kadhim M. and Miller F. (2014): Crouch gait changes after planovalgus foot deformity correction in ambulatory children with cerebral palsy. Gait \& Posture; 39: 793-798.

2. Rodda JM, Graham HK, Carson L, et al (2004): Sagittal gait patterns in spastic diplegia. J Bone Joint Surg Br; 86:251-8.

3. Rodda JM, Graham HK, Nattrass GR, et al (2006):Correction of Severe Crouch Gait in Patients with Spastic Diplegia with Use of Multilevel Orthopaedic Surgery, J Bone Joint Surg Am; 88: 2653-64.

4. Stout J, Gage R, Novacheck T, et al (2008): Distal Femoral Extension Osteotomy and Patellar Tendon Advancement to Treat Persistent Crouch Gait in Cerebral Palsy. $J$ Bone Joint Surg Am; 90: 2470-84.

5. Filho M, Neves D, Abreu F, et al (2008): Treatment of fixed knee flexion deformity and crouch gait using distal femur extension osteotomy in cerebral palsy. J Child Orthop; 2: 37-43.

6. Sossai R, Vavken P, Brunner R, et al (2015): Patellar tendon shortening for flexed knee gait in spastic diplegia. Gait \& Posture; 41: 658-665.

7. Das S, Pradhan S, Ganesh S, et al (2012): Supracondylar femoral extension osteotomy and patellar tendon advancement in the management of persistent crouch gait in cerebral palsy. Indian Journal of Orthopaedics; 46: 221-228.

8. Palisano RJ, Rosenbaum P, Bartlett D, et al. (2008): Content validity of the expanded and revised Gross Motor Function Classification System, Dev Med Child Neurol; 50:744750 .

9. Russell J, Rosenbaum L, Avery M, et al. (2002): Gross Motor Function Measure (GMFM-66 and GMFM-88) User's Manual. London, United Kingdom, Mac Keith Press .

10. Stout $T$ and Sugimoto K. (1989): New measurement of patellar height in the knees 
of children using the epiphyseal line midpoint, J Pediatr Orthop; 9:216-8.

11. Paley D. (2002): Osteotomy Concepts and Frontal Plane Realignment. Principles of Deformity Correction, Springer Berlin Heidelberg; 99-154

12. Novacheck TF, Stout JL, Gage JR, et al. (2009): Distal Femoral Extension Osteotomy and Patellar Tendon Advancement to Treat Persistent Crouch Gait in Cerebral Palsy Surgical Technique, Bone Joint Surg Am.; 91 Suppl 2:271-86.

13. Temelli Y and Akalan NE (2009): Treatment approaches to flexion contractures of the knee, Acta Orthop Traumatol Turc; 43:113120.

14. Gage JR. (1990): Surgical treatment of knee dysfunction in cerebral palsy, Clin Orthop Relat Res; 253:45-54.

15. Westberry DE, Davids JR, Jacobs JM, et al. (2006): Effectiveness of serial stretch casting for resistant or recurrent knee flexion contractures following hamstring lengthening in children with cerebral palsy, $J$ Pediatr Orthop; 26(1):109-14.

16. Beals RK. (2001): Treatment of knee contracture in cerebral palsy by hamstring lengthening, posterior capsulotomy and quadriceps mechanism shortening, Dev Med Child NeuroI; 43(12):802-5.

17. Novacheck TF, Stout JL, Gage JR, et al. (2009): Distal Femoral Extension Osteotomy and Patellar Tendon Advancement to Treat Persistent Crouch Gait in Cerebral Palsy Surgical Technique, Bone Joint Surg Am.; 91 Suppl 2:271-86.

18. Rutz E, Gaston MS, Camathias C, et al. (2012): Distal femoral osteotomy using the LCP pediatric condylar 90-degree plate in patients with neuromuscular disorders, $J$ Pediatr Orthop; 32(3):295-300.

19. Gavriliu S, Georgescu I, Ulici A, et al. (2013): Herbert capsuloplasty and Burnei tenomyoplasty for the correction of genu flexum in cerebral palsy, arthrogryposis and post-traumatic, Chirurgia 108(6):866-73.

20. Young JL, Rodda J, Selber P, et al. (2010): Management of the knee in spastic diplegia: what is the dose?, Orthop Clin North Am; 41(4):561-77.

21. Klatt J and Stevens PM. (2008): Guided growth for fixed knee flexion deformity, $J$ Pediatr Orthop; 28(6):626-31.

22. Healy MT, Schwartz MH, Stout JL, et al. (2011): Is simultaneous hamstring lengthening necessary when performing distal femoral extension osteotomy and patellar tendon advancement? Gait Posture; 33:1-5.

23. Joseph B, Reddy K, Varghese RA, et al. (2010): Management of severe crouch gait in children and adolescents with cerebral palsy, J Pediatr Orthop; 30(8):832-9.

24. El-Sobky TA, Fayyad TA, Kotb AM, et al. (2018): Bony reconstruction of hip in cerebral palsy children Gross Motor Function Classification System levels III to V: a systematic review, J Pediatr Orthop B; 27(3):221-230.

25. de-Souza RC, Mansano MV, Bovo M, et al. (2015): Hip salvage surgery in cerebral palsy cases: a systematic review, Rev Bras Ortop; 50(3):254-9.

26. Amen J, ElGebeily M, El-Mikkawy DM, et al. (2018): Single-event multilevel surgery for crouching cerebral palsy children: Correlations with quality of life and functional mobility, J Musculoskelet Surg Res; 2:148-55. 


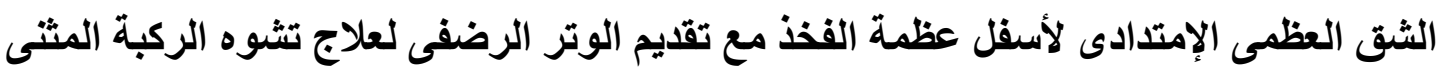

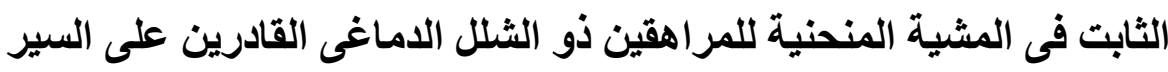

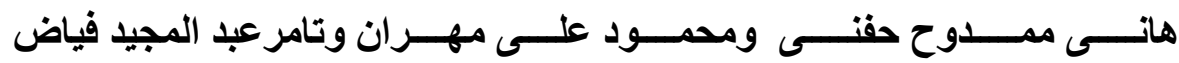

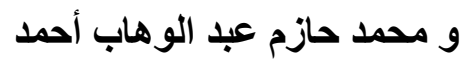

المقدمة: بعانى الأطفال المصابون بالثلل الدماغى والقادرون على الحركة من أنماط مختلفة من المشية الغير طيعية

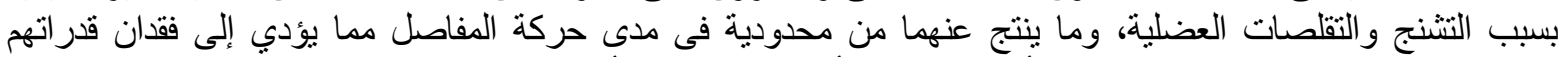

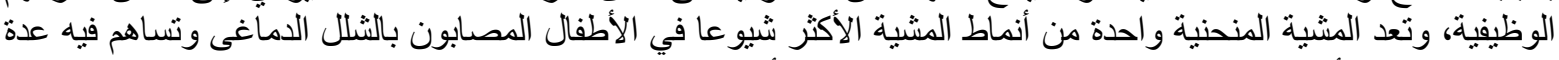

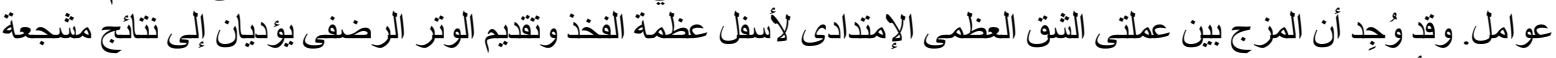
فى تقليل الألم وتحسين المشية.

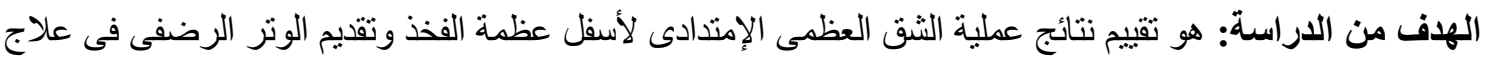

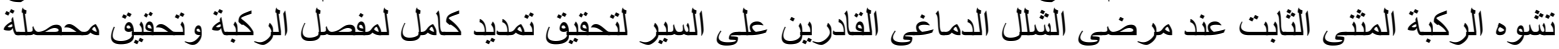

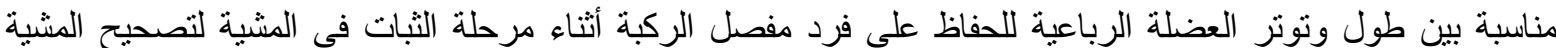

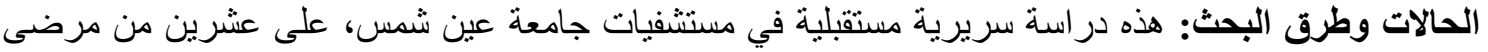

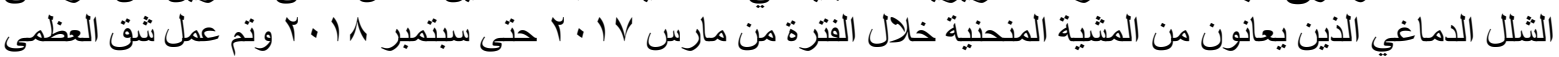

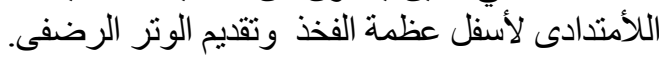

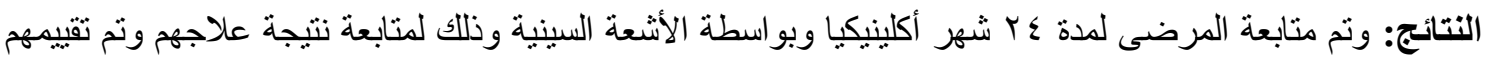

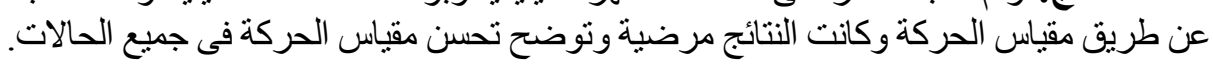

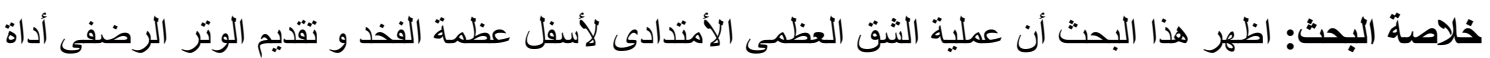
فعالة فى علاج تثوة الركبة المثىى الثابت فى حالات الثنلل الدماغى للمر اهقين القادرين على السير. 